ARTICLE

Received 20 Nov 2013 | Accepted 27 Feb 2014 | Published 24 Mar 2014

DOI: $10.1038 /$ ncomms 4522

\title{
Condensation of two-dimensional oxide-interfacial charges into one-dimensional electron chains by the misfit-dislocation strain field
}

\author{
C.-P. Chang ${ }^{1,2}$, M.-W. Chu1,3, H.T. Jeng ${ }^{4,5}$, S.-L. Cheng ${ }^{1,2}$, J.G. Lin'1, J.-R. Yang ${ }^{2}$ \& C.H. Chen ${ }^{1}$
}

The success of semiconductor technology is largely ascribed to controlled impacts of strains and defects on the two-dimensional interfacial charges. Interfacial charges also appear in oxide heterojunctions such as $\mathrm{LaAlO}_{3} / \mathrm{SrTiO}_{3}$ and $\left(\mathrm{Nd}_{0.35} \mathrm{Sr}_{0.65}\right) \mathrm{MnO}_{3} / \mathrm{SrTiO}_{3}$. How the localized strain field of one-dimensional misfit dislocations, defects resulting from the intrinsic misfit strains, would affect the extended oxide-interfacial charges is intriguing and remains unresolved. Here we show the atomic-scale observation of one-dimensional electron chains formed in $\left(\mathrm{Nd}_{0.35} \mathrm{Sr}_{0.65}\right) \mathrm{MnO}_{3} / \mathrm{SrTiO}_{3}$ by the condensation of characteristic twodimensional interfacial charges into the strain field of periodically arrayed misfit dislocations, using chemical mapping and quantification by scanning transmission electron microscopy. The strain-relaxed inter-dislocation regions are readily charge depleted, otherwise decorated by the pristine charges, and the corresponding total-energy calculations unravel the undocumented charge-reservoir role played by the dislocation-strain field. This two-dimensionalto-one-dimensional electronic condensation represents a novel electronic-inhomogeneity mechanism at oxide interfaces and could stimulate further studies of one-dimensional electron density in oxide heterostructures.

\footnotetext{
${ }^{1}$ Center for Condensed Matter Sciences, National Taiwan University, Taipei 106, Taiwan. ${ }^{2}$ Department of Materials Science and Engineering, National Taiwan University, Taipei 106, Taiwan. ${ }^{3}$ Taiwan Consortium of Emergent Crystalline Materials, Ministry of Science and Technology, Taipei 106, Taiwan. ${ }^{4}$ Department of Physics, National Tsing Hua University, Hsinchu 300, Taiwan. ${ }^{5}$ Institute of Physics, Academia Sinica, Taipei 115, Taiwan. Correspondence and requests for materials should be addressed to M.-W.C. (email: chumingwen@ntu.edu.tw) or to H.T.J. (email: jeng@phys.nthu.edu.tw).
} 
n epitaxial heterojunctions, the generic film-substrate misfit strains can be relaxed by one-dimensional (1D) misfit dislocations, forming semi-coherent interfaces characterized by strained dislocation cores with typically few nanometres in diameter and inter-dislocation epitaxial regions with vanishing strains ${ }^{1-5}$. The epitaxial strains can also be accommodated by uniform deformations of unit cells throughout the films, mediating coherent interfaces primitively free from the dislocation defects ${ }^{1-5}$.

The geometrical simplicity of coherent interfaces provides an optimal platform for tackling the pristine effect of strains on $2 \mathrm{D}$ interfacial charges in oxide heterostructures ${ }^{6,7}$. Indeed, it has been demonstrated that the coherent biaxial compressive/tensile strains can tailor the metallic $2 \mathrm{D}$ electron gas (2DEG) at the $\mathrm{LaAlO}_{3} /$ $\mathrm{SrTiO}_{3}(\mathrm{STO})$ interface ${ }^{6}$ and switch the $2 \mathrm{D}$ interfacial conductivity of the $R \mathrm{TiO}_{3} /$ STO heterostructures ( $R$, rare-earth elements $)^{7}$.

In comparison, effect of the localized dislocation-strain field on the functionality of oxide heterojunctions was much less studied and has, so far, centred on the investigation of ferroelectricity ${ }^{3,8-10}$, indicating a degradation of nominal spontaneous polarizations by the notable structural distortion within the strain-field range. The dislocation-strain impact on the 2D oxide-interfacial states like the above 2DEG in $\mathrm{LaAlO}_{3} / \mathrm{STO}^{6,11-13}$ and the localized 2D electron density in $\left(\mathrm{Nd}_{0.35} \mathrm{Sr}_{0.65}\right) \mathrm{MnO}_{3}(\mathrm{NSMO}) / \mathrm{STO}^{14}$ remains otherwise uninvestigated due to the challenging requirement of resolving the structural, chemical and electronic characteristics of the individual dislocations simultaneously at atomic-level precision ${ }^{13}$.

Using atomic-scale chemical mapping and quantification by scanning transmission electron microscopy (STEM) in conjunction with electron energy-loss spectroscopy (EELS) ${ }^{14,15}$, we report the observation of $2 \mathrm{D}$ interfacial-charge condensation into $1 \mathrm{D}$ electron chains by the misfit-dislocation strain field across the NSMO/STO interface. It was demonstrated recently that STEMEELS can count the oxide-interfacial charges at the atomic-planeby-atomic-plane, unit-cell-by-unit-cell level, determining the characteristic 2D interfacial charges of the epitaxial, dislocationfree NSMO/STO at atomic accuracy $\left(\sim 3.75 \times 10^{13} \mathrm{~cm}^{-2}\right)^{14,15}$. The STEM-EELS method thus enables an unambiguous examination of individual dislocations. The ever-measured charge density by the STEM-EELS renders the NSMO/STO a model system towards the understanding of interplay between misfit-dislocation strain fields and 2D interfacial charges.

\section{Results}

Imaging the dislocation-strain field. Figure 1a shows the STEM high-angle annular dark-field (HAADF) image of the NSMO/ STO (film thickness, $\sim 40 \mathrm{~nm}$ ) with the NSMO subject to nominally $\sim 2.7 \%$ of tensile strain ( $a=3.797 \AA, c=3.8925 \AA^{14}$; $a_{\mathrm{STO}}=3.905 \AA$ ). The bright (dark) NSMO film (STO substrate) arises from the atomic weight sensitivity of HAADF ${ }^{15-17}$, and misfit dislocations can be observed at the interface of this thick NSMO heterostructure as the regularly spaced dot-like contrasts (white arrows, Fig. 1a; average spacing, $\sim 30 \mathrm{~nm})^{4,18}$. In Fig. 1a (inset), the low-angle ADF (LAADF) imaging, a classical approach for revealing dislocation-strain contrasts ${ }^{5,19}$, resolves the characteristic strain field of the dislocations (arrows). The close HAADF inspection of an individual dislocation (Fig. 1b; blowup of the white square, Fig. 1a) unveils the NSMO and STO distortions by this dislocation-strain field, as observed by the tilting (deformation) of the unit cells along $a$ axis ( $c$ axis) with $\mathrm{A}$ and $\mathrm{B}$ circles denoting an unit cell $\left(\mathrm{ABO}_{3}\right.$, the general perovskite formula; $\mathrm{A}, \mathrm{Nd}$ and $\mathrm{Sr} ; \mathrm{B}, \mathrm{Ti}$ and $\mathrm{Mn}$ ).

Chemical and electronic tackling of the misfit dislocations. Figure $1 \mathrm{c}-\mathrm{f}$ shows the STEM-EELS chemical mapping summed over the results acquired at three individual dislocations exploiting the background-subtracted EELS intensities at $\mathrm{Nd}-\mathrm{N}_{2,3}$ ( $\sim 223 \mathrm{eV}, 4-\mathrm{eV}$ integration window), $\mathrm{Sr}_{-} \mathrm{M}_{3}(\sim 271 \mathrm{eV}, 4-\mathrm{eV})$, $\mathrm{Ti}_{2} \mathrm{~L}_{2}(\sim 466 \mathrm{eV}, 2-\mathrm{eV})$ and $\mathrm{Mn}-\mathrm{L}_{3}(\sim 642 \mathrm{eV}, 2-\mathrm{eV})$, respectively. The agreement between the chemical contrast maxima (Fig. 1c-f) and the reference atomic columns (Fig. 1b; see the guiding A and $\mathrm{B}$ circles, Fig. 1b-f) demonstrates that atomic resolution was systematically obtained in each map, unprecedented for the spatially resolved investigations of dislocations ${ }^{17-24}$. Intriguingly, the $\mathrm{Mn}-\mathrm{Ti}$ interdiffusion shows a radial pattern centred at the dislocation (see the dashed arcs, Fig. 1e,f) and nicely coincident with the dislocation-strain field revealed by the LAADF. The strain field of dislocations has been known to entangle with impurity segregation considering the associated cation-size mismatch (for example, $\mathrm{Mn}^{3.65+}, 0.57 \AA \mathrm{Mn}^{3+}, 0.645 \AA$; $\left.\mathrm{Mn}^{4+}, 0.53 \AA ; \mathrm{Ti}^{3+}, 0.67 \AA ; \mathrm{Ti}^{4+}, 0.605\right)$ would assist on the strain accommodation $2,9,17,25,26$. The radial interdiffusion pattern (Fig. 1e,f) can readily be an affirmation of the dislocation-strain field imaged by the $\operatorname{LAADF}^{9}$ and has intriguing correlations shortly.

Figure 2 exhibits the direct electronic inspections across the three individual dislocations investigated in Fig. 1c-f and also the interface of the six epitaxial inter-dislocation regions studied in Fig. 1i (red square, Fig. 1a; an exemplification of the regions with systematically $\sim 14 \sim 15 \mathrm{~nm}$ from the neighbouring dislocations). Surprisingly, the Mn- $L$ EELS spectra across the dislocations show a visible red shift, $\sim 1 \mathrm{eV}$, from N5 to S1-S2 (Fig. 2a; IF, the interfacial unit cell; N1 (S1), the first NSMO (STO) unit cell next to IF; N2-4 and S2, likewise), in distinct contrast to the robust $\mathrm{Mn}-L$ in the inter-dislocation regions (Fig. 2b). In manganese oxides, the chemical shift from $\mathrm{Mn}^{4+}$ to $\mathrm{Mn}^{3+}$ is characterized by a typical red shift of $\sim 1.5 \mathrm{eV}^{27}$. Considering also the linear chemical-shift scaling for the manganites ${ }^{27}$, the red shift of $\sim 1 \mathrm{eV}$ in Fig. 2a would correspond to a chemical shift from the nominal $\mathrm{Mn}^{3.65+}(\mathrm{N} 5)$ to $\mathrm{Mn}^{3}+(\mathrm{S} 1-\mathrm{S} 2)$, indeed in agreement with the peak position of the $\mathrm{Mn}^{3+}$ reference. The Ti- $L$ spectra across the dislocations also manifest a chemical shift as shown in Table 1, unlike basically $\mathrm{Ti}^{4+}$ character throughout the interdislocation regions (Fig. 2c). The misfit dislocations are obviously electron doped, but not the case for the inter-dislocation regions. In the dislocation-free NSMO/STO, it is, however, known that there exist characteristic interfacial charges throughout the interface $^{14}$. The misfit-dislocations visibly affect the pristine distribution of the characteristic 2D interfacial charges and this problem was further examined from stoichiometric aspects through Figs $1 \mathrm{c}-\mathrm{i}$ and 3.

Sub-stoichiometric misfit dislocations. At the misfit dislocations, chemical sub-stoichiometry appears as indicated by the weaker chemical contrasts around the dislocation in A-site maps (Fig. 1c,d) and the Mn contrast fluctuation across the dislocation in Fig. 1g, where the intensities of peaks 2 and 4 and the two grey Gaussians constituting peak 3 (that is, the dislocation) are weaker than those of peaks 1 and 5 . We have integrated the A-site (B-site) chemical contrasts in Fig. 1c,d (Fig. 1e,f) along $a$ axis to derive the overall sub-stoichiometry statistically. The thusobtained A- and B-site contrasts as shown in Fig. 3 (solid lines) were then summed, leading to the black A-site and grey B-site curves in Fig. 1h, respectively. For comparison, the stoichiometric evaluation like Fig. 1h was also conducted for the epitaxial interdislocation regions, giving rise to Fig. $1 \mathrm{i}$ that represents the atomic-plane-by-atomic-plane compositional variation across the interface ${ }^{14,15}$. In Fig. 1i, the attainment of unity at both the $\mathrm{A}$ and $\mathrm{B}$ sites reflects the nominal $\mathrm{ABO}_{3}$ therein ${ }^{14}$. The deficiency to 1 at each atomic plane in N3-S1 (Fig. 1h; grey N1, the dislocation) 
a
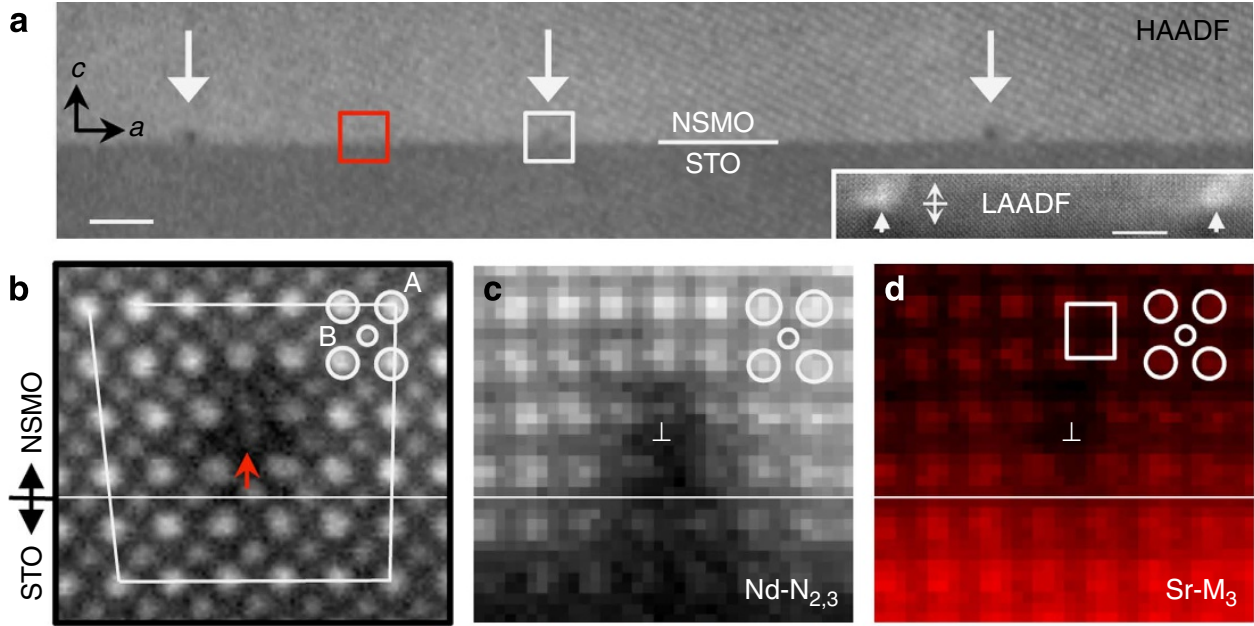

e
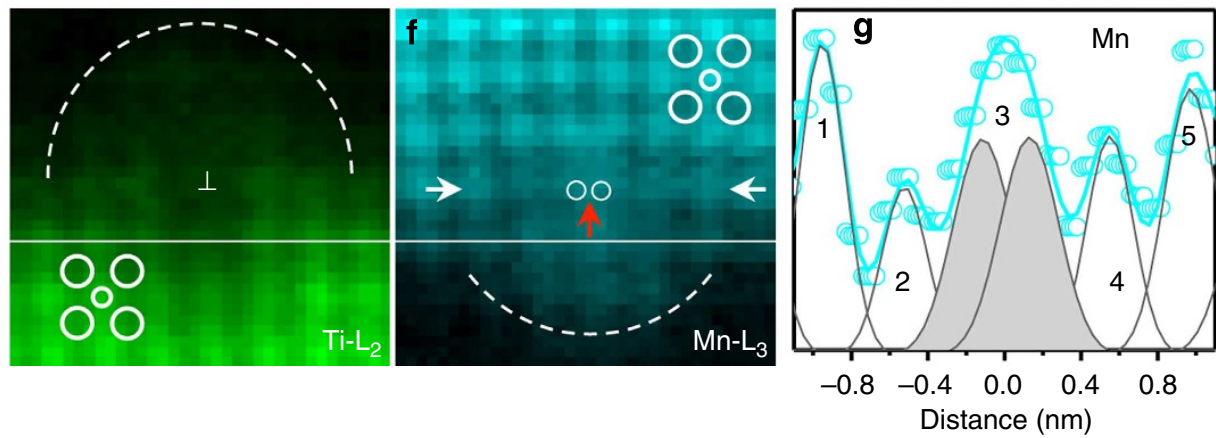

h

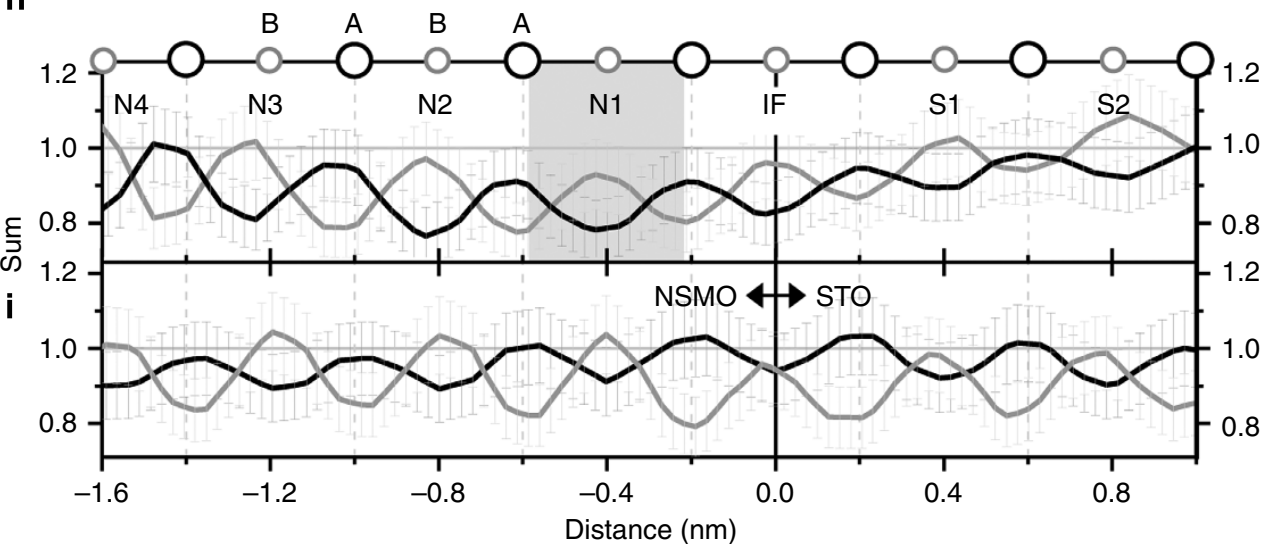

Figure 1 | STEM imaging and STEM-EELS chemical mapping and quantification. (a) The STEM-HAADF image of the NSMO/STO. White arrows, misfit dislocations. Red square, the exemplification of an inter-dislocation region. Inset, the STEM-LAADF image revealing the strain field of the dislocations (white arrows). Scale bar, $5 \mathrm{~nm}$. (b) The blowup of the individual dislocation indicated by the white square in (a). Red arrow, the dislocation. White open circuit, the Burgers circuit. The top-left open corner of the Burgers circuit determines the Burgers vector with a unit-cell length of $a<100>$, typical for edge-type misfit dislocations at perovskite-heterostructure interfaces. The oxygen is invisible due to its weak scattering power. (c-f) The respective Nd, Sr, $\mathrm{Ti}$ and $\mathrm{Mn}$ chemical maps ( $\perp$, the dislocation), obtained over the sum of three sets of results from three individual dislocations in different sample regions. The white rectangle in (d) signifies the unit-cell deformation along $\mathrm{c}$ axis in the dislocation cores. Dashed arcs in (e,f), the radial Ti-Mn intermixing pattern centred at the dislocation. (g) The Mn contrast profile across the dislocation in (f) (red arrow) along the double-arrowed lateral region therein. Circles, experimental results; solid lines, Gaussian-fitted profiles. At the dislocation (peak 3), the broad line width arises from the two constituent grey Gaussians, which correspond to two B-site atomic columns separated by $\sim 2.5 \AA$. (h) The unit-cell-by-unit-cell, atomic-plane-by-atomic-plane stoichiometric variations across the dislocation at N1. (i) The compositional characteristics across the NSMO/STO interface integrated over six interdislocation regions like the red square in (a). Black curve, the A-site ( $\mathrm{Nd}$ and $\mathrm{Sr}$ ) results. Grey curve, the B-site ( $\mathrm{Ti}$ and $\mathrm{Mn}$ ) results. In (h,i), 10\% error bars have been considered.

then signifies the sub-stoichiometry of the dislocation cores, resulting in $\sim 7 \%$ of average sub-stoichiometry for both the $\mathrm{A}$ and B. In the framework of charge neutrality, this average deficiency would correspond to a hole doping by +0.42 , suggesting a tendency towards $\sim \mathrm{Mn}^{4+}$ on the electronic examination across the dislocations $(\mathrm{Mn}$, the only element in the NSMO/STO eligible for accommodating the hole). In the corresponding Fig. 2a, we, however, observed the electron-doped feature at odds with the charge-neutrality consideration. Unambiguously, the dislocation cores are charged, and the 

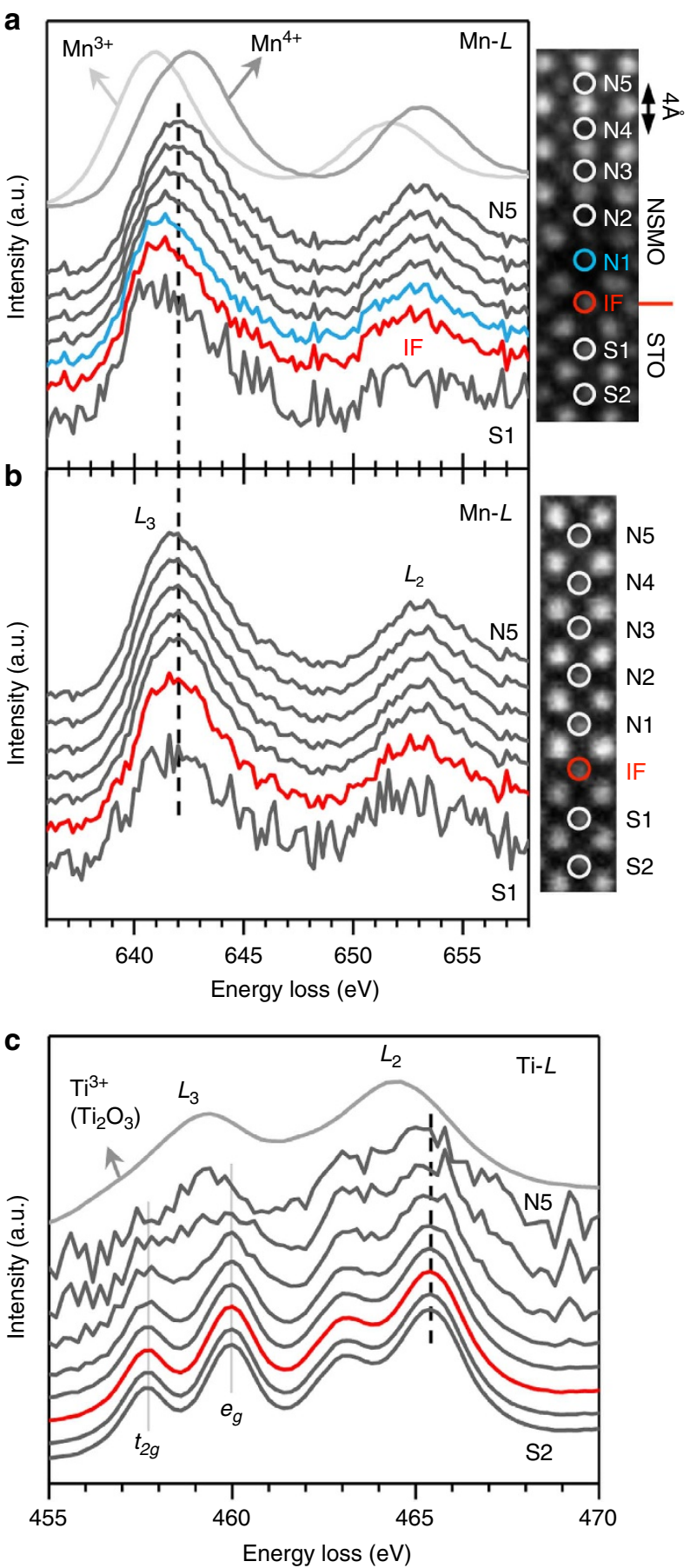

Figure 2 | Atomic-scale electronic inspections by STEM-EELS. (a) The $M n-L$ spectra acquired across the dislocation (blue N1) with each probed column signified in the right panel. The spectra are the sum over the three dislocations in Fig. 1c-f. The IF and S1-S2 spectra were directly acquired on the B-site, and each individual N1-N5 spectrum is effectively an average over the two neighbouring B-site columns. The reference $\mathrm{Mn}^{3+}$ and $\mathrm{Mn}^{4+}$ spectra were acquired on commercially available $\mathrm{Mn}_{2} \mathrm{O}_{3}$ and $\mathrm{MnO}_{2}$, respectively. (b,c) The respective $M n$ - and Ti- $L$ spectra acquired across the interface (red IF) of the inter-dislocation regions in Fig. 1i. The Mn- $L$ spectra at atomic-column S2 in $(\mathbf{a}, \mathbf{b})$ are noisy, whereas basically compatible with those at S1, thus not shown for simplicity. The dashed line in $(\mathbf{a}, \mathbf{b})$ points out the $\mathrm{Mn}$ chemical shift in the dislocation cores. The dashed line in (c) reveals the basically uniform $\mathrm{Ti}^{4+}$ state throughout the probed columns in the interdislocation regions. $t_{2 g}-e_{g}$ the characteristic crystal-field splitting of $\mathrm{Ti}^{4+}$. The reference $\mathrm{Ti}^{3+}$ spectrum was acquired on commercially available $\mathrm{Ti}_{2} \mathrm{O}_{3}$.
Table 1 | Cell-specific charge characteristics across the dislocation located at the $\mathbf{N} 1$ cell.

\begin{tabular}{lccc} 
Core cell & $\begin{array}{c}\text { A-site total } \\
\text { valence }\end{array}$ & $\begin{array}{c}\text { B-site total valence } \\
\text { (Mn/Ti valence*) }\end{array}$ & $\begin{array}{c}\text { Net charge } \\
\text { per core cell }\end{array}$ \\
\hline N4 & 2.35 & $3.59(+3.6 /+3.5)$ & -0.06 \\
N3 & 2.34 & $3.56(+3.5 /+3.7)$ & -0.10 \\
N2 & 2.31 & $3.51(+3.4 /+3.8)$ & -0.18 \\
N1 & 2.25 & $3.50(+3.2 /+3.9)$ & -0.25 \\
IF & 2.17 & $3.60(+3.1 /+3.9)$ & -0.23 \\
S1 & 2.09 & $3.79(+3.0 /+4.0)$ & -0.12 \\
S2 & 2.03 & $3.92(+3.0 /+4.0)$ & -0.05
\end{tabular}

IF, interfacial unit cell.

*The individual $\mathrm{Mn}$ valence was determined by the classical approach of linearly interpolating each $\mathrm{Mn}$ peak in Fig. $2 \mathrm{a}$ to the $\mathrm{Mn}^{4+}-\mathrm{Mn}^{3+}$ chemical shift of $1.5 \mathrm{eV}^{27}$. The individual Ti valence was estimated by the generally accepted method of a linear least-square fitting with the respective $\mathrm{Ti}^{4+}$ and $\mathrm{Ti}^{3+}$ spectra of $\mathrm{STO}$ and $\mathrm{Ti}_{2} \mathrm{O}_{3}$ as the references ${ }^{14}$. In addition to these $\mathrm{B}$-site valences, the composition of each core cell is also required upon the cell-specific charge estimation ${ }^{14}$ and can be derived from the respective $\mathrm{Nd}, \mathrm{Sr}, \mathrm{Ti}$ and $\mathrm{Mn}$ fractions in Fig. 3. The sub-stoichiometric N3-S1 cells were then normalized to the nominal $\mathrm{ABO}_{3}$, characteristic of $\mathrm{N} 4$ and $\mathrm{S} 2$, for the convenience of the evaluation of the corresponding charge detail as tabulated above.

cation deficiency in Fig. $1 \mathrm{~h}$ and the impurity intermixing in Fig. 1e,f are not the predominant factors to this feature. The charged cores are otherwise scalable through the electron-doping level in each N4-S2 (Table 1), leading to the unit-cell-by-unit-cell charge examination in Fig. 4a,b.

1D electron chains and total-energy examinations. Imposing now the strain field in Fig. 1e,f on Fig. 4a (dashed circle), we surprisingly found that the strain-field radius of $\sim 1.2 \mathrm{~nm}$ (Fig. 4a) is notably consistent with the charge-distribution range of $\sim 1.2 \mathrm{~nm}$ from the dislocation (blue curve, Fig. $4 \mathrm{~b}$; see also the grey guiding lines). The inter-dislocation regions are, by contrast, charge neutral (red curve) and the dislocation charges spread over $\mathrm{N} 4-\mathrm{S} 2$ with $\sim 0.14 \mathrm{e} /$ core cell in average (Fig. $4 \mathrm{~b}$ ). Comparing this average charge with the characteristic $2 \mathrm{D}$ charges of the dislocation-free NSMO/STO $\left(\sim 3.75 \times 10^{13} \mathrm{~cm}^{-2}\right)$ by integrating the charges within the strain field and redistributing them along the interface (Methods), we obtain the 2D analogy of $\sim 3.3 \times 10^{13} \mathrm{~cm}^{-2}$, in remarkable consistency with the pristine density. Clearly, the characteristic 2D interfacial charges segregate into the strain field of the $1 \mathrm{D}$ dislocations, forming $1 \mathrm{D}$ electron chains. These unbalanced charges could give rise to a local electrostatic potential as estimated in Fig. $4 \mathrm{c}(\sim 0.03 \mathrm{eV} \text {; Methods })^{23}$.

Such a 2D-to-1D electronic condensation has not been observed in dislocation studies before ${ }^{8,9,18-25,28}$, and we performed a microscopic inspection on this intriguing straincharge correlation by total-energy calculations as shown in Fig. 5. The inset exhibits a coherently strained NSMO (tensile, 2.7\%) with $a b$-plane clamped to STO and $c$ axis readily compressed in the constant-volume context, resulting in the reduced tetragonality $(c / a)$ of $\sim 0.942$ compared with the nominal $\sim 1.025$ (vertical dashed line). The corresponding total energy (horizontal zero line, Fig. 5) forms the basis for evaluating the strain-charge entanglement. On electron doping to the chargeneutral, coherently strained NSMO (see the red curve in Fig. 5, $0.025 e$ per unit cell of doping; blue, 0.05; green, 0.075), the respective total energies at $c / a \sim 0.942$ drastically increase as a result of the ever increasing electrostatic cost for such unbalanced charges in the NSMO. More specifically, the electrostatic cost can be as significant as $\sim 0.23 \mathrm{eV}$ per unit cell on the doping level of $0.05 e$ per unit cell (blue, Fig. 5), much larger than the estimated electrostatic potential of $\sim 0.03 \mathrm{eV}$ (Fig. $4 \mathrm{c} ; \sim 0.024 \mathrm{eV}$ in the total-energy calculations, Methods) and rendering the doped cells less favourable. This latter feature is correlated with the fact that 


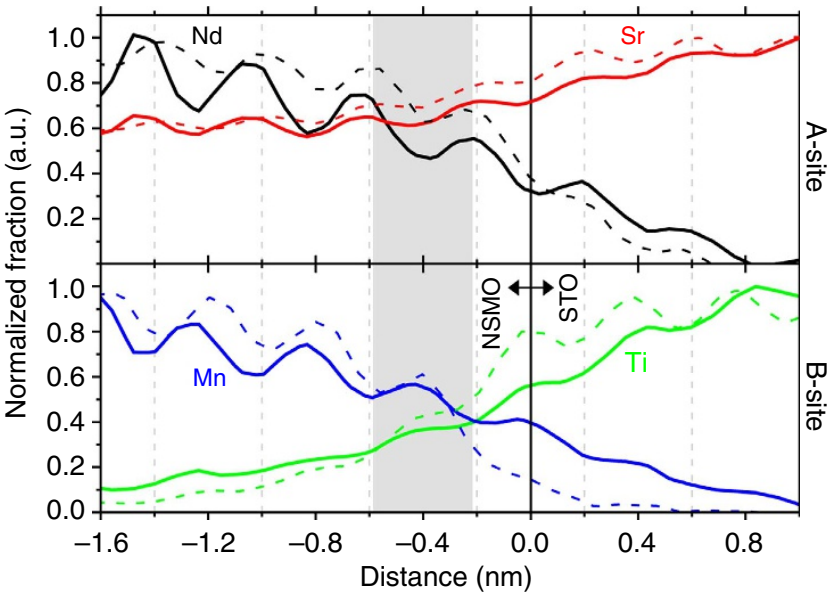

Figure 3 | Chemical profiles in the NSMO/STO. The element-specific chemical profiles across the three misfit dislocations studied in Fig. 1c- $f$ (solid) and the interface of the six inter-dislocation regions in Fig. 1i (dashed). Grey area, the dislocation location. The profiles were obtained by integrating each chemical map of the dislocations and the inter-dislocation regions along $a$ axis. The thus-obtained profiles were then normalized to the chemical contrasts of the unit cell furthest away from the NSMO/STO interface at $0 \mathrm{~nm}$. A statistical error of $10 \%$ like that in Fig. 1h,i has been omitted for the clarity of the current presentation. In the classical context of strain-relaxed semi-coherent interfaces, a dislocation spacing of $b / \gamma(b$, the length of the Burgers vector; $\gamma$, the misfit strain; thus $\sim 14 \sim 15 \mathrm{~nm}$ for the $\mathrm{NSMO} / \mathrm{STO}$ ) can be expected ${ }^{4}$, while we observed $\sim 30 \mathrm{~nm}$, suggesting that the chemical intermixing observed can be of an origin for mitigating the characteristic epitaxial strain by the corresponding cation-size mismatch ${ }^{2,5,25}$. A larger dislocation spacing than anticipated readily arises.

the characteristic 2D interfacial charges in the dislocation-free $\mathrm{NSMO/STO}$ have to be accommodated by the Ti impurity in the $\mathrm{NSMO}^{14}$. To further capture the essence behind these energetic subtleties, we numerically increase $c$ axis of the coherently strained NSMO that simulates the $c$-deformed NSMO in the dislocation cores with a tetragonality of $\sim 1.02 \sim 1.03$ (that is, grey area in Fig. 5) as exemplified by the white rectangle in Fig. 1d. Remarkably, the introduction of such a structural distortion in the NSMO compensates for the electrostatic cost of doping, lowering the total energy and allowing an electron-doping level of $\sim 0.05 \sim 0.052 e$ per unit-cell, as revealed in Fig. 5 by the crossing of the blue-curve valley through zero in the grey area that conceptually depicts the strained dislocation cores. Most importantly, this thus-derived doping level, equivalent to $\sim 3.2 \times 10^{13} \mathrm{~cm}^{-2}$ in $2 \mathrm{D}$, is notably consistent with that observed in Fig. $4 \mathrm{~b}$ and conforming to a reasonable energy cost for the charge condensation into the dislocation-strain field. In Fig. 5, an energy gain of $\sim 0.23 \mathrm{eV}$ per unit cell is indeed suggested on the charge accommodation by the corresponding $c$-strained NSMO. Moreover, Fig. 5 also reveals that a charge-neutral state is most favourable for the epitaxial NSMO (black curve), consistent with the charge depletion observed in the inter-dislocation regions (Fig. 4b). The emergence of strained dislocation cores at the interface thus becomes a convenient solution for hosting the generic interfacial charges, leading to the observed $1 \mathrm{D}$ electron chains periodically arrayed at the interface (Figs 1a and 4a,b).

Oxygen vacancies. Figure $6 \mathrm{a}, \mathrm{b}$, respectively, displays the $\mathrm{O}-\mathrm{K}$ EELS spectra taken by positioning the electron probe at N5-S2 across the misfit dislocations and the interface of the inter-

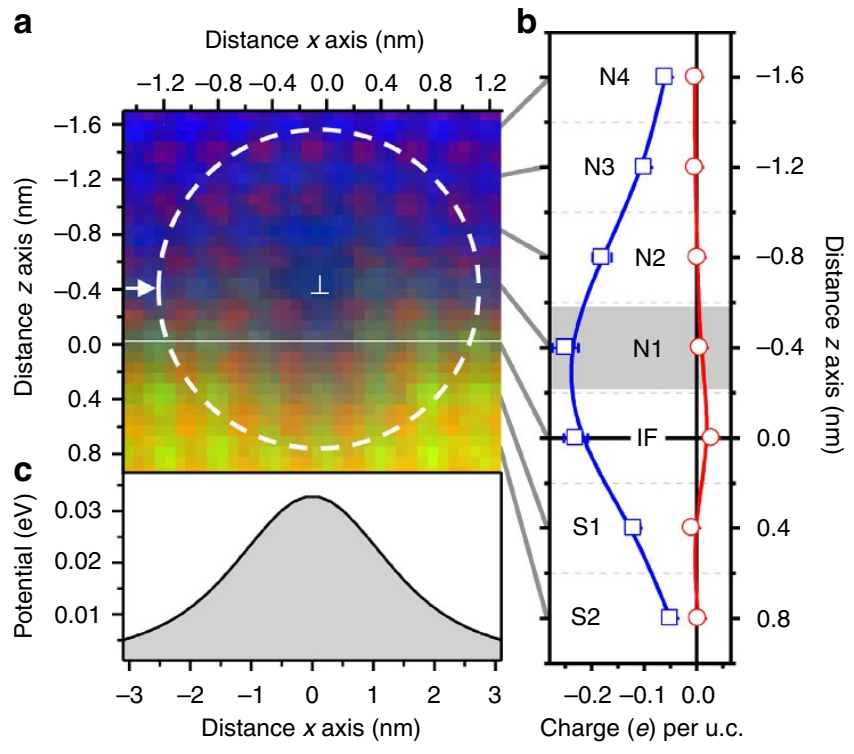

Figure 4 | Condensation of the 2D interfacial charges into 1D electron chains by the misfit-dislocation strain field. (a) The superposition of the dislocation-strain field (dashed circle; derived from Fig. 1e,f) with the Sr-Ti$\mathrm{Mn}$ overlaid chemical map (red, Sr; green, Ti; blue, $\mathrm{Mn}$ ), revealing the radial strain field with a radius of $\sim 1.2 \mathrm{~nm}$ from the dislocation $(\perp)$. (b) The charge-distribution pattern in the dislocation cores (blue curve; grey N1, the dislocation), showing a distribution range of $\sim 1.2 \mathrm{~nm}$ in consistence with the strain-field radius in (a). Red curve, the unit-cell-by-unit-cell charge characteristics in the inter-dislocation regions (u.c.: unit cell). $10 \%$ of statistical errors have been incorporated into both curves. (c) The estimated electrostatic potential across the dislocation at $0 \mathrm{~nm}$, derived along the white-arrowed direction in (a).

dislocation regions shown in Fig. 2. In general, all spectra consist of four features, peaks A-D. The calculated partial density of states (DOS) including also the effect of oxygen vacancies are shown in Fig. 6c (black, no oxygen vacancy; red, one oxygen vacancy per $\mathrm{MnO}_{6}$ octahedron). The calculations were performed in the framework of $\mathrm{Nd}_{0.25} \mathrm{Sr}_{0.75} \mathrm{MnO}_{3}$, which represents the most convenient composition for incorporating the oxygen vacancy on a reasonable calculation cost. For convenience, $\mathrm{Nd}_{0.25} \mathrm{Sr}_{0.75} \mathrm{MnO}_{3}$ hereby is termed as NSMO, since the corresponding total DOS, Fig. 6d, show no significant difference to those of $\mathrm{Nd}_{0.33} \mathrm{Sr}_{0.67} \mathrm{MnO}_{3}$ that faithfully captures the physical essence of the nominal NSMO (Methods) ${ }^{14}$. Comparing all spectra in Fig. 6a with those in Fig. $6 \mathrm{~b}$ reveals that the dislocation cores are characterized by distinct spectral changes in peaks A and B throughout the N5-S2 core cells. The previous investigations of the $\mathrm{O}-\mathrm{K}$ spectra of STO have indicated that such fine-structure changes can be the consequence of oxygen vacancies ${ }^{29,30}$. For NSMO (for example, N1-N5), the peak A is predominated by the $\mathrm{Mn}-3 d$ states and the peak B is a convolution of both the Mn-3d and Nd-5d states (see Fig. $6 c, d$ ). The electronic influence of oxygen vacancies on the Mn-3d states was examined in Fig. $6 \mathrm{c}$ (red curve), where the introduced vacancy indeed affects the corresponding partial DOS in consistence with the associated spectral change in Fig. 6a. Oxygen vacancies should exist in the dislocation cores. The influence of A-site (B-site) vacancies on the $\mathrm{O}-K$ spectra was also investigated in our calculations and found to principally affect the overall spectral weight of peaks $B-D$ $(\mathrm{A}-\mathrm{B})$, in agreement with previous studies on the subject ${ }^{24,29}$ and also our observations in Fig. 6a. However, the notable structural distortions in the dislocation cores might also play a role in the spectral difference between Fig. $6 a$ and $b$. 


\section{Discussion}

Charged dislocations in oxides have attracted continuous interests due to the remarkable character of local electronic inhomogeneities, which are conventionally ascribed to chemical sub(off)stoichiometry arising from vacancies and impurities that are helpful for strain accommodations in the vicinity of dislocation

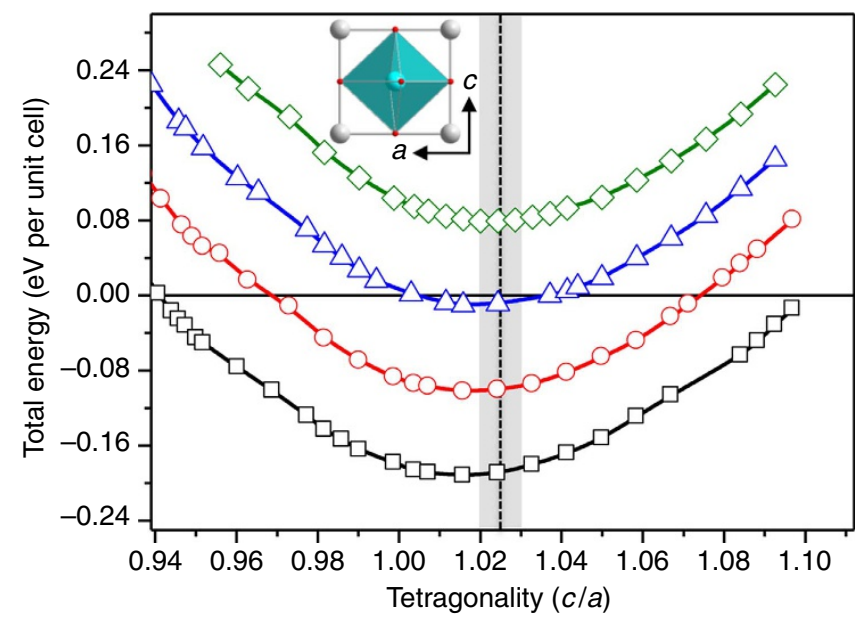

Figure 5 | The theoretical examination of the strain-charge entanglement addressing the 2D-to-1D electron condensation in the NSMO. The

total-energy evolution of a tensile-strained NSMO (inset) as a function of various electron-doping levels (red, 0.025 e per unit cell; blue, 0.05; green, 0.075 ) and increasing strains as scaled by the increasing tetragonality (c/a). Black curve, the calculations for the charge-neutral NSMO. The dashed line, the nominal tetragonality of $\sim 1.025$. The grey region, the estimated tetragonality of the distorted NSMO in the dislocation cores (see the white rectangle in Fig. 1d). cores $^{8,9,18-25,28}$. Herein, vacancy and impurity segregations in the cores are indeed observed (Figs $1 \mathrm{~h}, 3$ and 6). Nonetheless, the experimental and theoretical elaborations at the atomic scale suggest that the charged character is not dictated by these atomic disorders, but rather by a remarkable strain-driven condensation of the 2D charges into $1 \mathrm{D}$ electron chains. This thus-derived charge-reservoir role of the dislocation-strain field is totally undocumented before and represents a novel form of electronic inhomogeneities at oxide interfaces. Indeed, it was not found until recently that the $2 \mathrm{DEG}$ in respective $\mathrm{LaAlO}_{3} /$ and $\mathrm{LaTiO}_{3} / \mathrm{STO}$ heterostructures is electronically inhomogeneous and readily shows the surprising coexistence of magnetic order and superconductivity ${ }^{31-37}$. Furthermore, it was demonstrated that 1D-like striped electronic inhomogeneities with a notably enhanced carrier density can exist in the $\mathrm{LaAlO}_{3} / \mathrm{STO}$ due to the presence of striped domain boundaries at the interface as a result of the tetragonal phase transition of STO below $\sim 105 \mathrm{~K}^{37-40}$. These very recent breakthroughs have intensified the search for further electronic inhomogeneities in oxide heterojunctions through the studies of unique lowdimensional structures formed within the 2D electronic states $^{37,40}$, such as misfit dislocations reported here and interfacial structural kinks theoretically proposed ${ }^{41}$. It should be noted that in literatures there exists only one report on the effect of $1 \mathrm{D}$ dislocations on 2D oxide-interfacial charges conducted on $\mathrm{LaAlO}_{3} / \mathrm{STO}^{13}$. In the work, the dislocations were purposely introduced so as to be perpendicular to the interface and penetrating throughout the heterostructure ${ }^{13}$, unlike the misfit dislocations naturally formed at interfaces in our case. A conclusion of charge-depleted discs surrounding the line defects has been deduced ${ }^{13}$, dissimilar to the 2D-to-1D electron condensation we observed. A future STEM-EELS investigation of $\mathrm{LaAlO}_{3} / \mathrm{STO}$ with misfit dislocations systematically at the interface would be essential.
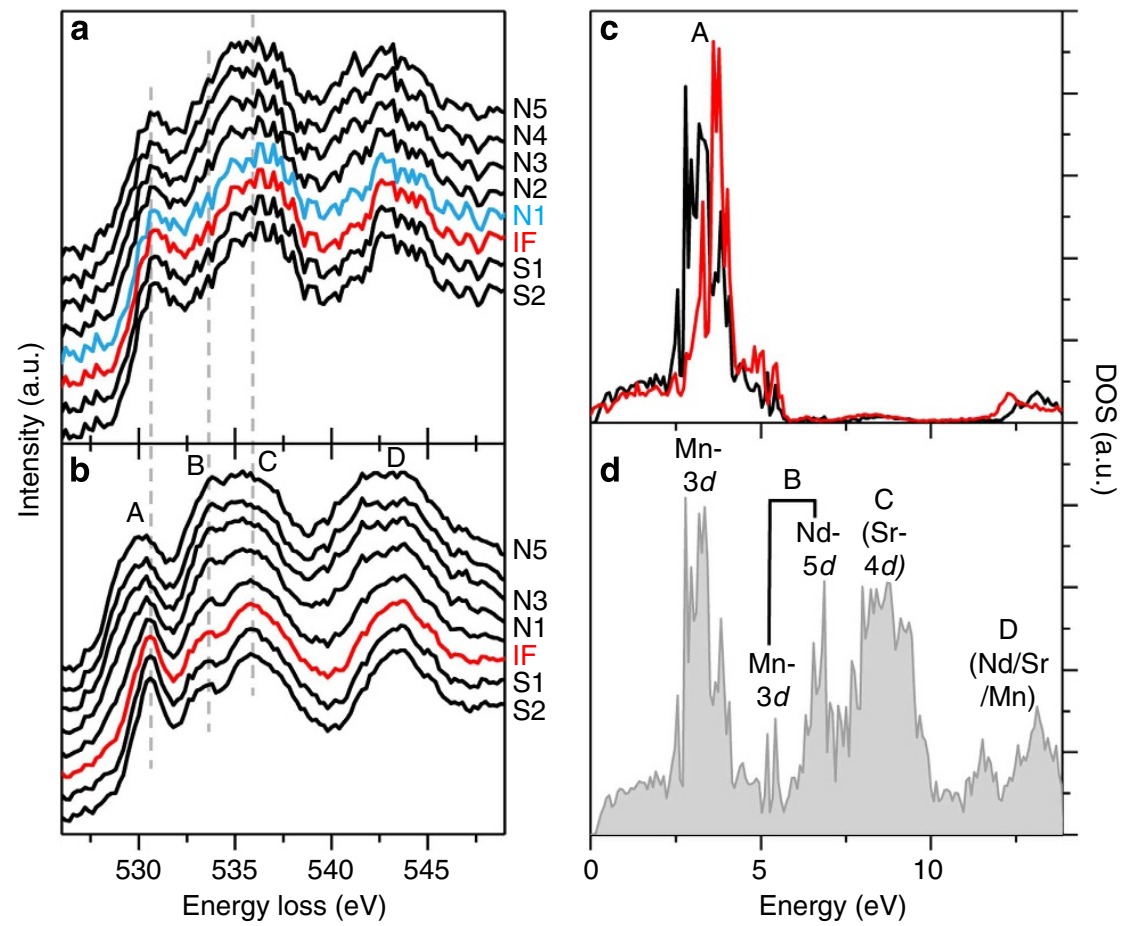

Figure 6 | Oxygen $\boldsymbol{K}$-edge EELS spectra and electronic calculations. The oxygen $K$-edge spectra acquired at the dislocation cores, (a), and in the inter-dislocation regions, (b), on scanning the electron probe across the dislocation (N1, a) and the interface (IF, b) as indicated in Fig. 2. Vertical dashed lines, guides for the eyes. Peaks A-D, see text. (c) the calculated Mn partial DOS of nominal (black) and oxygen-deficient NSMO (red; see also text). (d) the total DOS of NSMO. 
The physics of 2D oxide-interfacial charges is continuously evolving ${ }^{31-37}$, whereas the $1 \mathrm{D}$ counterpart is just dawning ${ }^{37-40}$, with the primary task focusing on the innovative approaches for the engineering of $1 \mathrm{D}$ electronic states $37,40,41$. It is anticipated that our stimulating observation of $1 \mathrm{D}$ electron chains by the condensation of $2 \mathrm{D}$ interfacial charges into the misfit-dislocation strain field would have far-reaching repercussions in these emerging explorations of $1 \mathrm{D}$ electron density and related phenomena at oxide interfaces.

\section{Methods}

Material growth and STEM-EELS characterizations. The [001]-oriented NSMO/ STO heterostructure was grown on $\mathrm{TiO}_{2}$-terminated STO using pulsed laser deposition and the STEM-EELS chemical mapping and quantification were conducted on an aberration-corrected electron microscope (JEOL-2100F) ${ }^{14}$. The specimen thickness along the incident probe direction is $\sim 30 \mathrm{~nm}$, and the HAADF and LAADF collection angles are $70 \sim 187$ and 14 37 mrad, respectively. An EELS collection angle of $\sim 30 \mathrm{mrad}$ and a probe convergent angle of $\sim 20 \mathrm{mrad}$ were exploited with a corresponding probe size (current) of $\sim 1.2 \AA(\sim 120 \mathrm{pA})$. All STEM-EELS results have been subject to careful principal component analysis for removing the spectral random noises without introducing artefacts and then spectral background removals ${ }^{14}$.

Charge and potential evaluations. To obtain the $2 \mathrm{D}$ equivalence of the charges segregated into the dislocation-strain field, we integrated the charges therein and homogeneously redistributed them along the interface by exploiting the associated formula of $\pi R^{2} \rho / d e$, where $R$ is the charge-distribution radius $(\sim 1.2 \mathrm{~nm}$, Fig. $4 \mathrm{a}, \mathrm{b})$, $\rho$ is the electron density per unit core-cell volume $(\sim 0.14 e$ per core cell; volume $\left.\sim 64 \AA^{3}\right), d$ is the average dislocation spacing $(\sim 30 \mathrm{~nm}$, Fig. 1a) and $e$ is the unit electron charge ${ }^{23}$. Owing to the charge condensation into 1D electron chains observed in Fig. 4 b, an electrostatic potential $(\phi)$ would build up at the dislocation and can be evaluated in the framework of Thomas-Fermi screening, most suitable for perovskite oxides, through the following established formulation ${ }^{23}$.

$$
\phi(x)=\frac{S e}{2 \varepsilon \varepsilon_{0} k_{0}} e^{-k_{0}|x|}
$$

where $S$ is the $2 \mathrm{D}$ charge density evaluated above, $x$ is the distance from the dislocation $(0 \mathrm{~nm})$ along the white arrow in Fig. $4 \mathrm{a}, \varepsilon_{0}(\varepsilon)$ is the permittivity of vacuum (dielectric constant; $\sim 181$, an average between

$\mathrm{NSMO} \sim(\mathrm{La}, \mathrm{Sr}) \mathrm{MnO}_{3} \sim 30$ and STO $\left.\sim 332\right)$ and $k_{0}=l_{\mathrm{TF}}^{-1}$ with $l_{\mathrm{TF}}$ being the Thomas-Fermi screening length $\left(l_{\mathrm{TF}}=\sqrt{\pi \varepsilon a_{0} / 4\left(3 \pi^{2} \rho\right)^{1 / 3}}\right.$ with the Bohr radius $\left(a_{0}\right)$ of $0.53 \AA$ and thus-derived $l_{\mathrm{TF}}$ of $\left.\sim 1.37 \mathrm{~nm}\right)^{23,42}$. A Lorenzian line-shape fitting was then applied to the evaluated position $(x)$-dependent potential for smoothing the drastic exponential decay described by the formulation, resulting in Fig. 4c. It is noted that the potential height is notably small, $\sim 0.03 \mathrm{eV}$ (Fig. 4c), comparable to the room-temperature thermal agitation of $\sim 0.026 \mathrm{eV}$.

Theoretical total-energy calculations. The total-energy calculations were conducted on the basis of $\mathrm{Nd}_{0.33} \mathrm{Sr}_{0.67} \mathrm{MnO}_{3}$, formally shown to capture the essence of the NSMO with an otherwise reasonably sized supercell of $\sqrt{2} a \times \sqrt{ } 2 a \times 3 c$ (ref. 14). All calculations have been performed in the VASP package using the fullpotential augmented wave method within the generalized gradient approximation plus an on-site Hubbard repulsion $(U)$ of $5 \mathrm{eV}$. The structural feature of each calculated point in Fig. 5 has been optimized by a residual atomic force of $<0.04 \mathrm{eV}^{-1}$ and all the calculations were carried out over the $5 \times 5 \times 3$ Monkhorst-Pack $k$-mesh with the energy cutoff of $400 \mathrm{eV}$. The total energy shown in Fig. 5 is a result of dividing that of the supercell by a factor of six (that is, the supercell size) to reveal the total energy of an actual NSMO unit cell. The onset of an electrostatic potential in the $1 \mathrm{D}$ electron chains due to the unbalanced charges was also intrinsically incorporated in the calculations through the effective potential exploited, including a thorough consideration over electron-nucleus attraction, electron-electron coulomb repulsion, on-site correlation $U$ and exchange-correlation potential. For the unbalanced charges of $0.05 e$ per unit cell in the optimally deformed NSMO (Fig. 5), an electrostatic potential of $\sim 0.024 \mathrm{eV}$ has been thus derived, notably close to $\sim 0.03 \mathrm{eV}$, in the classical electrostatic evaluation and also one order of magnitude smaller than the electrostatic cost of $\sim 0.23 \mathrm{eV}$ per unit cell upon the strain-charge entanglement described in the text.

\section{References}

1. Pantelides, S. T. The role of extended defects in device degradation. Phys. Status Solidi A 210, 175-180 (2013).

2. Narayan, J. Recent progress in thin film epitaxy across the misfit scale. Acta Mater. 61, 2703-2724 (2013).

3. Chu, M.-W. et al. Impact of misfit dislocations on the polarization instability of epitaxial nanostructured ferroelectric perovskites. Nat. Mater. 3, 87-90 (2004).
4. Matthews, J. W. \& Blakeslee, A. E. Defects in epitaxial multilayers: I. Misfit dislocations. J. Cryst. Growth 27, 118-125 (1974).

5. Pennycook, S. J. et al. Misfit accommodation in oxide thin film heterostructures. Acta Mater. 61, 2725-2733 (2013).

6. Bark, C. W. et al. Tailoring a two-dimensional electron gas at the $\mathrm{LaAlO}_{3} /$ $\mathrm{SrTiO}_{3}$ (001) interface by epitaxial strain. Proc. Natl Acad. Sci. USA 108, 4702-4724 (2011).

7. Jang, H. W. et al. Metallic and insulating oxide interfaces controlled by electronic correlations. Science 331, 886-889 (2011).

8. Jia, C. L. et al. Effect of a single dislocation in a heterostructure layer on the local polarization of a ferroelectric layer. Phys. Rev. Lett. 102, 117601 (2009).

9. Arredondo, M. et al. Direct evidence for cation non-stoichiometry and Cottrell atmospheres around dislocation cores in functional oxide interfaces. Adv. Mater. 22, 2430-2434 (2010).

10. Lubk, A. et al. Electromechanical coupling among edge dislocations, domain walls, and nanodomains in $\mathrm{BiFeO}_{3}$ revealed by unit-cell-wise strain and polarization maps. Nano Lett. 13, 1410-1415 (2013).

11. Mannhart, J. \& Schlom, D. G. Oxide interfaces-an opportunity for electronics. Science 327, 1607-1611 (2010).

12. Hwang, H. Y. et al. Emergent phenomena at oxide interfaces. Nat. Mater. 11, 103-113 (2012).

13. Thiel, S. et al. Electron scattering at dislocations in $\mathrm{LaAlO}_{3} / \mathrm{SrTiO}_{3}$ interfaces. Phys. Rev. Lett. 102, 046809 (2009).

14. Chang, C. P. et al. Atomic-scale observation of a graded polar discontinuity and a localized two-dimensional electron density at an insulating oxide interface. Phys. Rev. B 87, 075129 (2013).

15. Chu, M.-W. \& Chen, C. H. Chemical mapping and quantification at the atomic scale by scanning transmission electron microscopy. ACS Nano 7, 4700-4707 (2013).

16. Arslan, I., Bleloch, A., Stach, E. A. \& Browning, N. D. Atomic and electronic structure of mixed and partial dislocations in GaN. Phys. Rev. Lett. 94, 025504 (2005).

17. Buban, J. P. et al. Gain boundary strengthening in alumina by rare earth impurities. Science 311, 212-215 (2006).

18. Kurata, H., Isojima, S., Kawai, M., Shimakawa, Y. \& Isoda, S. Local analysis of the edge dislocation core in $\mathrm{BaTiO}_{3}$ thin film by STEM-EELS. J. Microsc. 236, 128-131 (2009).

19. Fitting, L., Thiel, S., Schmehl, A., Mannhart, J. \& Muller, D. A. Subtleties in $\mathrm{ADF}$ imaging and spatially resolved EELS: a case study of low-angle twist boundaries in $\mathrm{SrTiO}_{3}$. Ultramicroscopy 106, 1053-1061 (2006).

20. Batson, P. E., Kavanagh, K. L., Woodall, J. M. \& Mayer, J. W. Electron-energyloss scattering near a single misfit dislocation at the GaAs/GaInAs interface. Phys. Rev. Lett. 57, 2729-2732 (1986).

21. Batson, P. E. Atomic and electronic structure of a dissociated $60^{\circ}$ misfit dislocation in $\mathrm{Ge}_{x} \mathrm{Si}_{(1-x)}$. Phys. Rev. Lett. 83, 4409-4412 (1999).

22. Kim, M. et al. Non-stoichiometry and the electrical activity of grain boundaries in $\mathrm{SrTiO}_{3}$. Phys. Rev. Lett. 86, 4056-4059 (2001).

23. Klie, R. F., Beleggia, M., Zhu, Y., Buban, J. P. \& Browning, N. D. Atomic-scale model of the grain boundary potential in perovskite oxides. Phys. Rev. B 68, 214101 (2003).

24. Zhang, Z., Sigle, W. \& Rühle, M. Atomic and electronic characterization of the a[100] dislocation core in $\mathrm{SrTiO}_{3}$. Phys. Rev. B 66, 094108 (2002).

25. Klie, R. F. et al. Enhanced current transport at grain boundaries in high- $T_{c}$ superconductors. Nature 435, 475-478 (2005).

26. Shannon, R. D. Revised effective ionic radii and systematic studies of interatomic distances in halides and chalcogenides. Acta Cryst. A32, 751-767 (1976).

27. Chakhalian, J. et al. Orbital reconstruction and covalent bonding at an oxide interface. Science 318, 1114-1117 (2007).

28. McKenna, K. P. \& Shluger, A. L. Electron-trapping polycrystalline materials with negative electron affinity. Nat. Mater. 7, 859-862 (2008).

29. Mizoguchi, T. et al. vacancy segregation by heat treatment at $\mathrm{SrTiO}_{3}$ grain boundary. Appl. Phys. Lett. 87, 241920 (2005).

30. Muller, D. A., Nakagawa, N., Ohtomo, A., Grazul, J. L. \& Hwang, H. Y. Atomic scale imaging of nanoengineered oxygen vacancy profiles in $\mathrm{SrTiO}_{3}$. Nature 430, 657-661 (2004).

31. Ariando, et al. Electronic phase separation at the $\mathrm{LaAlO}_{3} / \mathrm{SrTiO}_{3}$ interface. Nat. Commun. 2, 188 (2011).

32. Li, L., Richter, C., Mannhart, J. \& Ashoori, R. C. Coexistence of magnetic order and two-dimensional superconductivity at $\mathrm{LaAlO}_{3} / \mathrm{SrTiO}_{3}$ interfaces. Nat. Phys. 7, 762-766 (2011).

33. Bert, J. A. et al. Direct imaging of the coexistence of ferromagnetism and superconductivity at the $\mathrm{LaAlO}_{3} / \mathrm{SrTiO}_{3}$ interface. Nat. Phys. 7, 767-771 (2011).

34. Dikin, D. A. et al. Coexistence of superconductivity and ferromagnetism in two dimensions. Phys. Rev. Lett. 107, 056802 (2011).

35. Mehta, M. et al. Evidence for charge-vortex duality at the $\mathrm{LaAlO}_{3} / \mathrm{SrTiO}_{3}$ interface. Nat. Commun. 3, 955 (2012). 
36. Biscaras, J. et al. Multiple quantum criticality in a two-dimensional superconductor. Nat. Mater. 12, 542-548 (2013).

37. Caprara, S. et al. Multiband superconductivity and nanoscale inhomogeneity at oxide interfaces. Phys. Rev. B 88, 020504(R) (2013).

38. Brinkman, A. Streaks of conduction. Nat. Mater. 12, 1085-1086 (2013).

39. Kalisky, B. et al. Locally enhanced conductivity due to the tetragonal domain structure in $\mathrm{LaAlO}_{3} / \mathrm{SrTiO}_{3}$ heterointerfaces. Nat. Mater. 12, 1091-1095 (2013).

40. Honig, M. et al. Local electrostatic imaging of striped domain order in $\mathrm{LaAlO}_{3} /$ $\mathrm{SrTiO}_{3}$. Nat. Mater. 12, 1112-1118 (2013).

41. Bristowe, N. C., Fix, T., Blamire, M. G., Littlewood, P. B. \& Artacho, E. Proposal of a one-dimensional electron gas in the steps at the $\mathrm{LaAlO}_{3}-\mathrm{SrTiO}_{3}$ interface. Phys. Rev. Lett. 108, 166802 (2012).

42. Hikita, Y., Nishikawa, M., Yajima, T. \& Hwang, H. Y. Termination control of the interface dipole in $\mathrm{La}_{0.7} \mathrm{Sr}_{0.3} \mathrm{MnO}_{3} / \mathrm{Nb}: \mathrm{SrTiO}_{3}$ (001) Schottky junctions. Phys. Rev. B 79, 073101 (2009).

\section{Acknowledgements}

We thank Prof. J.-J. Lin, Dr C.-W. Chang, Dr W.W. Pai, Dr. Y.-Y. Chin, Dr. H.-J. Lin and

Prof. W.F. Pong for enlightening discussions on the results. H.T.J. also thanks NCHC,
CINC-NTU and NCTS, Taiwan for technical supports. This work was supported by Ministry of Science and Technology, National Taiwan University, National Tsing Hua University and Academia Sinica Taiwan.

\section{Author contributions}

C.-P.C. and M.-W.C. conducted the experiments and wrote the paper. H.T.J. performed the theoretical calculations. S.-L.C. and J.G.L. grew the NSMO/STO heterostructure. J.-R.Y. characterized the structural characteristics of the misfit dislocations. C.H.C. supervised the work and imbued his ideas for interpretations of the results.

\section{Additional information}

Competing financial interests: The authors declare no competing financial interests.

Reprints and permission information is available online at http://npg.nature.com/ reprintsandpermissions/

How to cite this article: Chang, C.-P. et al. Condensation of two-dimensional oxideinterfacial charges into one-dimensional electron chains by the misfit-dislocation strain field. Nat. Commun. 5:3522 doi: 10.1038/ncomms4522 (2014). 